\title{
Development of Palmprint Verification System Using Biometrics*
}

\author{
G. Shobha ${ }^{+}$, M. Krishna, S.C. Sharma \\ (R.V. College of Engineering, Mysore Road, Bangalore 560059, India) \\ +Corresponding author: E-mail: shobhatilak@rediffmail.com
}

\author{
Shobha G, Krishna M, Sharma SC. Development of palmprint verification system using biometrics. Journal \\ of Software, 2006,17(8):1824-1836. http://www.jos.org.cn/1000-9825/17/1824.htm
}

\begin{abstract}
Palmprint verification system using Biometrics is one of the emerging technologies, which recognizes a person based on the principle lines, wrinkles and ridges on the surface of the palm. These line structures are stable and remain unchanged throughout the life of an individual. More importantly, no two palmprints from different individuals are the same, and normally people do not feel uneasy to have their palmprint images taken for testing. Therefore palmprint recognition offers a promising future for medium-security access control systems. In this paper, a new approach for personal authentication using hand images is discussed. Gray-Scale palm images are captured using a digital camera at a resolution of $640 \times 480$. Each of these gray-scale images is aligned and then used to extract palmprint and hand geometry features. These features are then used for authenticating users. The image acquisition setup used here is inherently simple and it does not employ any special illumination nor does it use any pegs that might cause any inconvenience to users. Experimental results show that the designed system achieves an acceptable level of performance.
\end{abstract}

Key words: gray scale image; image binarization; thersholding; boundary tracking; peak detection; features; normalization; hand geometry

\section{Introduction}

Reliability in personal authentication is key to the security in any transactional database. Many physiological characteristics of humans i.e., biometrics, are typically time invariant, easy to acquire, and unique for every individual. Biometric features such as face, iris, fingerprint, hand geometry, palmprint, signature, etc. have been suggested for the security in access control. Most of the current research in biometrics has been focused on fingerprint and face. The reliability of personal identification using face is currently low as the researchers today continue to grabble with the problems of pose, lighting, orientation and gesture. Finger print identification is widely used in personal identification as it works well in most cases. However it is difficult to acquire fingerprint features i.e. minutiae, for some class of characteristics which are receiving increasing attention. Moreover, additional biometric features, such as palmprints, can easily be integrated with the existing authentication system to provide an enhanced level of confidence in personal authentication. Palmprint is one of the relatively new physiological biometrics due to its stable and unique characteristics. The rich texture information of palmprint offers one of the powerful means in personal recognition. Biometric palmprint recognizes a person based on the principal lines, wrinkles and ridges on the surface of the palm. These line structures are stable and remain unchanged throughout the life of an individual. More importantly, no two palmprints from different individuals are the same, and normally

* Received 2004-07-20; Accepted 2005-07-08 
people do not feel uneasy to have their palmprint images taken for testing. Therefore palmprint recognition offers promising future for medium-security access control system. Palmprint verification system is a one-to-one matching process. It matches a person's claimed identity to an enrolled pattern. There are two phases in the system-enrollment and verification.

1. At the enrollment stage, a set of the template images represented by their extracted features is labeled and stored into a database.

2. At the verification stage, features are extracted from an input image, and then are matched with the claimant's palmprint image features stored in the database.

Both phases comprise the following steps: preprocessing for palmprint localization, enhancement and feature extraction. Figure 1 shows an abstract functional view of the proposed palmprint verification system.

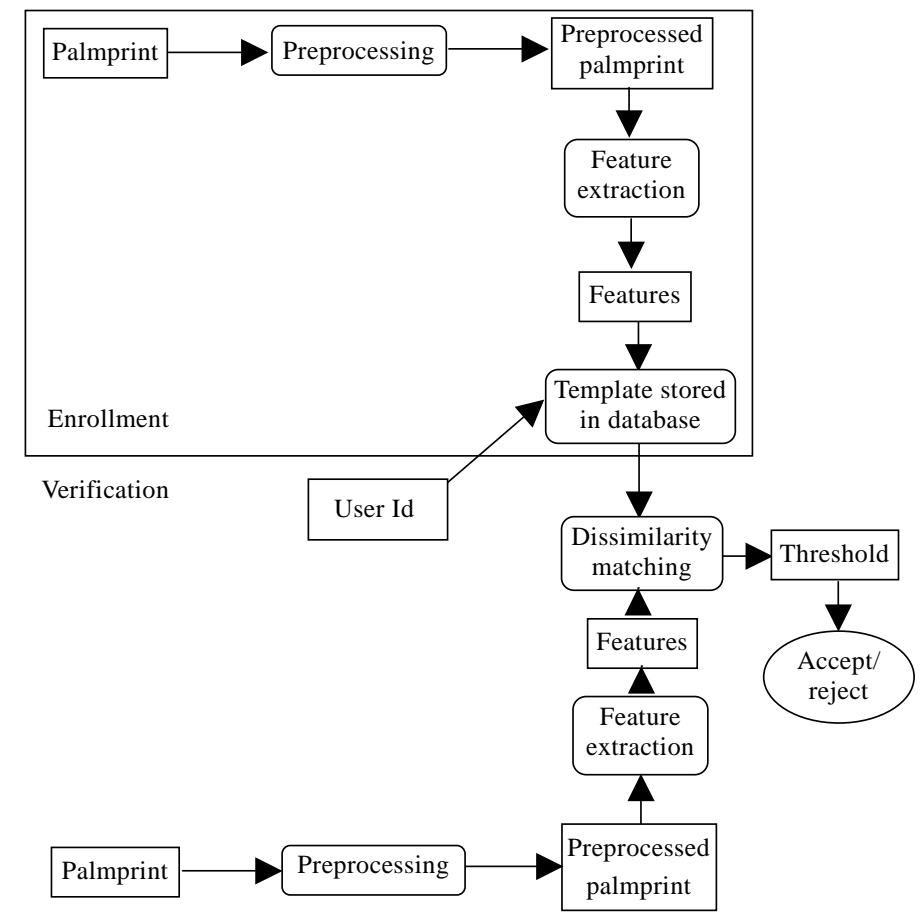

Fig.1 Palmprint verification system-Functional view

\section{Literature Survey}

\subsection{Priori work}

Two kinds of biometric indicators can be extracted from the low-resolution hand images:

- Palmprint features, which are composed of principal lines, wrinkles, minutiae etc., and

- Hand geometry features, which include area/size of palm, length and width of fingers.

The problem of personal verification using palmprint features has drawn considerable attention and researchers have proposed various methods. One popular approach considers palmprints as textured images which are unique to every individual. Therefore, analysis of palmprint images using Gabor filters ${ }^{[5]}$, wavelets ${ }^{[6]}$, Fourier transform ${ }^{[7]}$, and local texture energy ${ }^{[8]}$ has been proposed in the literature. The endpoints of some prominent principal lines, i.e., the heart-line, headline, and life-line are rotation invariant. Some authors ${ }^{[9,13]}$ have used these endpoints and midpoints for the registration of geometrical and structural features of principal lines for palmprint matching. Duta 
et $a l .^{[1]}$ have suggested that the connectivity of extracted palm lines is not important Therefore; they have used a set of feature points along the prominent palm lines, instead of extracted palm lines, to generate the matching score for palmprint authentication. The palmprint pattern also contains ridges and minutiae, similar to a fingerprint pattern. However, in palmprints the creases and ridges often overlap and cross each other.

Chen et $a l .{ }^{[10]}$ have attempted to estimate palmprint crease points by generating a local gray level directional map. These crease points are connected together to isolate the crease in the form of line segments, which are used in the matching process. Some related work on palmprint verification also appears in Refs.[11,12]. Conventional palmprint authentication methods utilize inked palmprint images while the recent work in Refs. [6,13] have shown the utility of inkless palmprint images acquired from the digital scanner. However, some promising results on the palmprint images acquired from image acquisition systems using CCD based digital camera appears in Refs.[5,11]. The US patent office has issued several patents for devices that measure hand geometry features for personal verification. Some related work using low-resolution digital hand images appears in Refs.[13,14]. These authors have used fixation pegs to restrict the hand movement and shown promising results. However, the results in Refs.[13,14] may be biased by the small size of the database and an impostor can easily violate the integrity of system by using fake hand.

\subsection{Proposed system}

The palmprint and hand geometry images can be extracted from a hand image in a single shot at the same time. Unlike other multi-biometrics systems (e.g., face and fingerprint, voice and face, etc.), user does not have to undergo the inconvenience of passing through multiple sensors. Block diagram of the proposed verification system is shown in Fig.2.

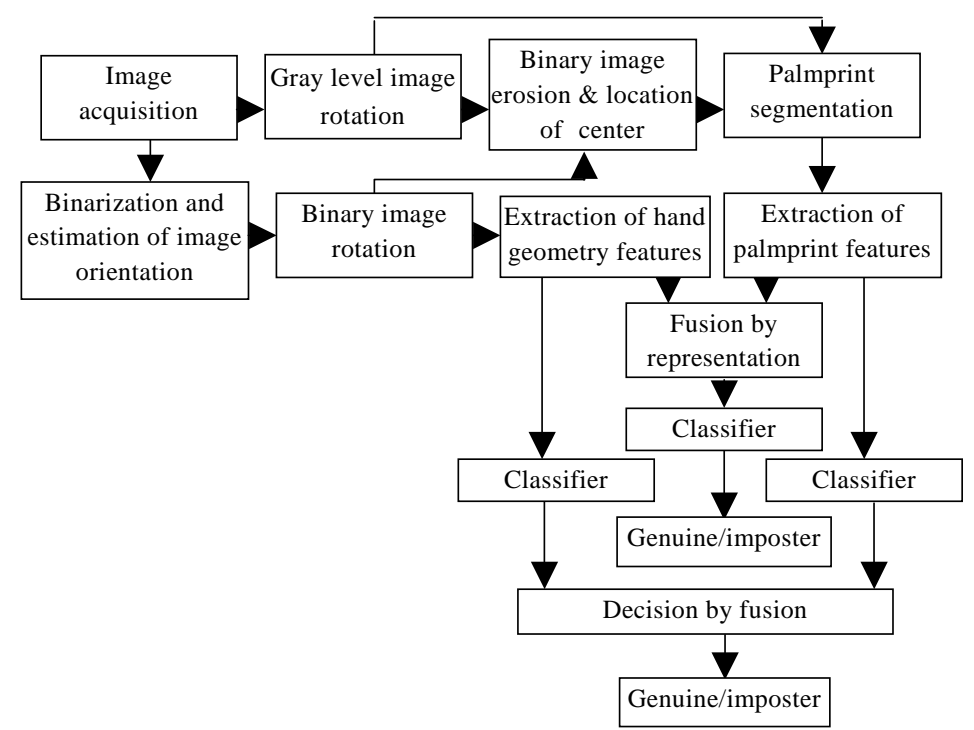

Fig.2 Palmprint verification system-Detailed view

Hand images of every user are used to automatically extract the palmprint and hand geometry features. This is achieved by first thresholding the images acquired from the digital camera. The resultant binary image is used to estimate the orientation of hand since in absence of pegs user does not necessarily align their hand in a preferred direction. The rotated binary image is used to compute hand geometry features. This image also serves to estimate the center of palmprint from the residue of morphological erosion with a known structuring element (SE). This center point 
is used to extract the palmprint image of a fixed size, from the rotated gray level hand images. Each of these palmprint images is used to extract salient features. Thus the palmprint and hand geometry features of an individual are obtained from the same hand image.

\section{Design Details of Palmprint Verification System (PVS)}

\subsection{Reading the image}

The image format used in PVS is the 256 gray-scale BMP format. This format stores images as a 2-D grid of pixel values, each pixel being represented by a byte. Here, the image bitmap is read into a 2-D double array and is interpreted using the file size. Figure 3 shows a grayscale BMP palm image parsed and rendered by palmprint verification system (PVS).

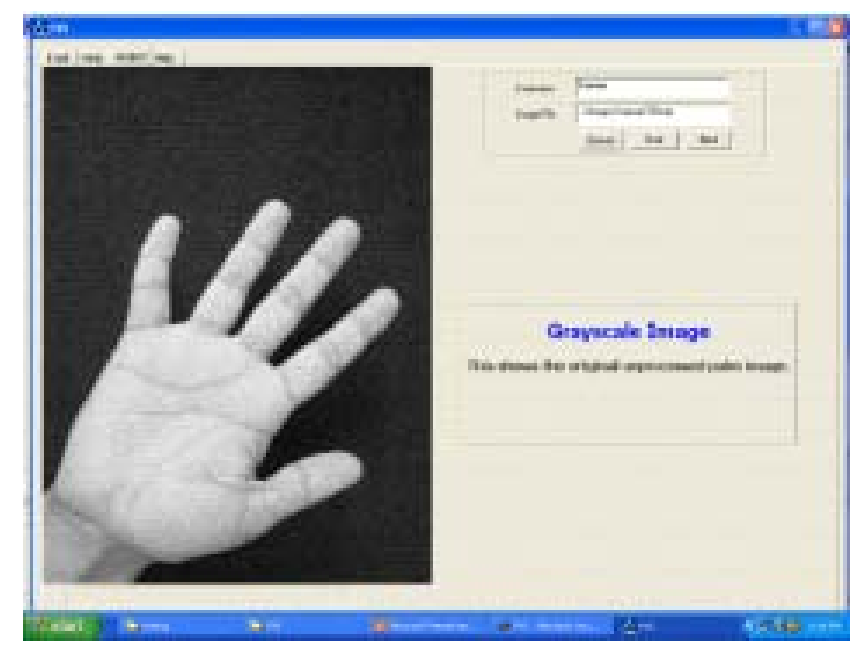

Fig.3 Gray-Scale palm image

\subsection{Image binarization/thresholding}

Segmentation involves separating an image into regions (or their contours) corresponding to objects. Usually regions are segmented by identifying common properties. Or, similarly, contours are identified by identifying differences between regions (edges).

The simplest property that pixels in a region can share is intensity. So, a natural way to segment such regions is through thresholding, the separation of light and dark regions. Thresholding creates binary images from gray-level ones by turning all pixels below some threshold to zero and all pixels about that threshold to one.

If $g(x, y)$ is a thresholded version of $f(x, y)$ at some global threshold $T$,

$$
g(x, y)= \begin{cases}1, & \text { if } f(x, y) \geq T \\ 0, & \text { otherwise }\end{cases}
$$

\subsubsection{Problems with thresholding}

The major problem with thresholding is that only the intensity is considered, not any relationships between the pixels. There is no guarantee that the pixels identified by the thresholding process are contiguous. Extraneous pixels that aren't part of the desired region can easily be included, and isolated pixels within the region (especially near the boundaries of the region) can be easily missed. These effects get worse as the noise gets worse, simply because it's more likely that pixels intensity doesn't represent the normal intensity in the region. When the thresholding is used, it results sometimes losing too much of the region and sometimes getting too many extraneous background pixels (Shadows of 
objects in the image are also a real pain-Not just where they fall across another object but where they mistakenly get included as part of a dark object on a light background).

\subsubsection{Otsu's clustering method}

One way of determining the optimal threshold ${ }^{[12]}$ is by trying to make each cluster as tight as possible, thus minimizing their overlap. Obviously, distributions can't be changed, but threshold can be adjusted. As threshold is adjusted one way, the spread of one can be increased and the other can be decreased. The goal then is to select the threshold that minimizes the combined spread.

The within-class variance $\sigma^{2}$ is defined as the weighted sum of the variances of each cluster

$$
\sigma_{\text {Within }}^{2}(T)=n_{B}(T) \sigma_{B}^{2}(T)+n_{O}(T) \sigma_{O}^{2}(T)
$$

where

$$
\begin{aligned}
& n_{B}(T)=\sum_{i=0}^{T-1} p(i) \\
& n_{O}(T)=\sum_{i=T}^{n-1} p(i)
\end{aligned}
$$

$$
\begin{aligned}
& \sigma_{B}^{2}(T)=\text { the variance of the pixels below threshold } \\
& \sigma_{O}^{2}(T)=\text { the variance of the pixels above threshold }
\end{aligned}
$$

and $[0, N-1]$ is the range of intensity levels.

Computing this within-class variance for each of the two classes for each possible threshold involves a lot of computation, but there's an easier way. If the within-class variance is subtracted from the total variance of the combined distribution, the between-class variance is obtained:

$$
\sigma_{\text {Between }}^{2}(T)=\sigma^{2}-\sigma_{\text {Within }}^{2}(T)=n_{B}(T)\left[\mu_{B}(T)-\mu\right]^{2}+n_{O}(T)\left[\mu_{O}(T)-\mu\right]^{2}
$$

where $\sigma^{2}$ is the combined variance and $\mu$ is the combined mean.

Substituting Eq.(7) and simplifying,

$$
\sigma_{\text {Between }}^{2}(T)=n_{B}(T) n_{O}(T)\left[\mu_{B}(T)-\mu_{O}(T)\right]^{2}
$$

So, for each potential threshold $T$,

1. Separate the pixels into two clusters according to the threshold;

2. Find the mean of each cluster;

3. Square the difference between the means;

4. Multiply the number of pixels in one cluster by the number in the other.

This depends only on the difference between the means of the two clusters, thus avoiding calculating differences between individual intensities and the cluster means. The optimal threshold is the one that maximizes the between-class variance (or, conversely, minimizes the within-class variance). This still sounds like a lot of work, since it is done for each possible threshold, but it turns out that the computations aren't independent from one threshold to another. The individual intensities and the respective cluster means can be updated as pixels move from one cluster to the other and as $T$ increases, using the following simple recurrence relations:

$$
\begin{gathered}
n_{B}(T+1)=n_{B}(T)+n_{T} \\
n_{O}(T+1)=n_{O}(T)-n_{T} \\
\mu_{B}(T+1)=\frac{\mu_{B}(T) n_{B}(T)+n_{T} T}{n_{B}(T+1)} \\
\mu_{O}(T+1)=\frac{\mu_{O}(T) n_{O}(T)+n_{T} T}{n_{O}(T+1)}
\end{gathered}
$$

Figure 4 shows a binary palm image obtained by thresholding the grayscale image with an optimal threshold 
chosen by the Otsu method.

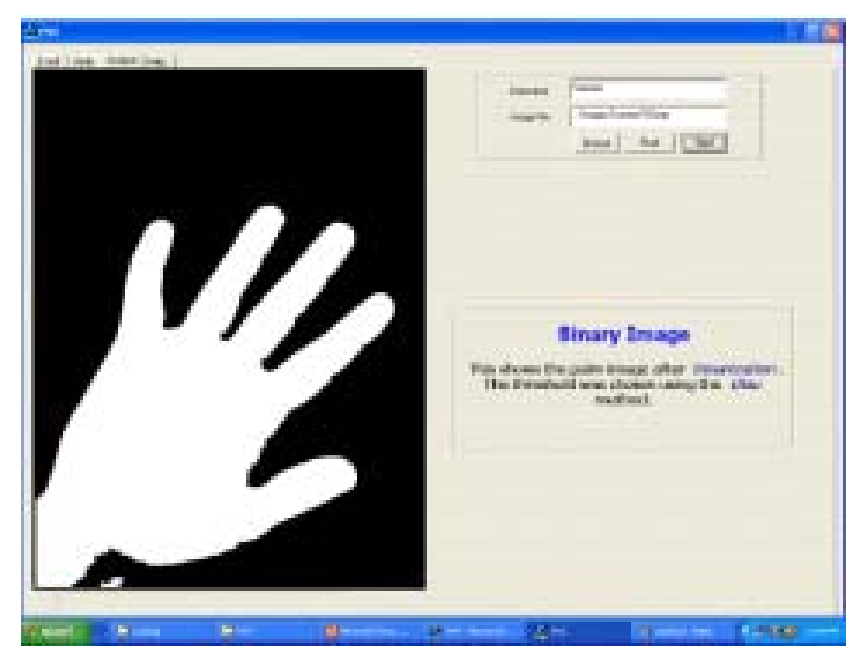

Fig.4 Binary palm image

\subsection{Morphological processing}

"Mathematical morphology" means using mathematical principals to manipulate shape ${ }^{[6]}$.

\subsubsection{Image regions as sets}

The basis of mathematical morphology is the description of image regions as sets. For a binary image, we can consider the "on" (1) pixels to comprise a set of values from the "universe" of pixels in the image. The "off" (0) pixels are thus the set compliment of the set of on pixels.

The standard set notation to describe image operations are described as follows:

$A$ : the image

$A^{c}$ : the compliment of the image (inverse)

$A \cup B$ : the union of images $A$ and $B$

$A \cap B$ : the intersection of images $A$ and $B$

$A-B=A \cap B^{c}$ : the difference between $A$ and $B$ (the pixels in $A$ that aren't in $B$ )

$\# A$ : the cardinality of $A$

The image A translated by movement vector $\mathrm{t}$ is

$$
A_{t}=\{c \mid c=a+t \text { for some } a \in A\}
$$

\subsubsection{Dilation and erosion}

Dilation is defined as follows ${ }^{[7]}$

$$
A \oplus B=\{c \mid c=a+t \text { for some } a \in A \text { and } b \in B\}
$$

One way to think of this is to take copies of $A$ and translate them by movement vectors defined by each of the pixels in $B$ (This means we have to define an origin for $B$ ). If we union these copies together.

We get $A \oplus B$

$$
A \oplus B=\bigcup_{t \in B} A_{t}
$$

Erosion is defined as follows

$$
A \ominus B=\{x \mid x+b \in A \text { for every } b \in B\}
$$

One way to think of this is to take copies of $A$ and again translate them by movement vectors defined by each 
of the pixels in $B$. However, this time we move them in the opposite direction $(-b)$ and intersect the copies together.

$$
A \ominus B=\bigcap_{t \in B} A_{-t}
$$

\subsubsection{Opening and closing}

An opening is erosion followed by dilation with the same structuring element ${ }^{[6]}$.

$$
A \circ B=(A \ominus B) \oplus B
$$

Since the erosion finds all the places where the structuring element fits inside the image, but it only marks these positions at the origin of the element. By following erosion by dilation, we "fill back in" the full structuring element at places where the element fits inside the object. So, an opening can be considered to be the union of all translated copies of the structuring element that can fit inside the object.

Openings can be used to remove small objects, protrusions from objects, and connections between objects. So, for example, opening by a $5 \times 5$ square element will remove all things that are less than 5 pixels high or less than 5 pixels tall.

$$
A \bullet B=(A \oplus B) \ominus B
$$

Closing works in an opposite fashion from opening.

\subsubsection{Boundary tracking and peak detection}

It is assumed that the objects consist of white pixels only, and the black pixels belong to the background. Let us first define the properties called 4-connectivity and 8-connectivity ${ }^{[10]}$. Two pixels are said to be 4-connected to each other if they are neighbors via any of the four cardinal directions $(N, E, S$, and $W$ ). Two pixels are said to be 8-connected if they are neighbors via any of the eight directions $(N, N E, E, S E, S, S W, W$, and $N W)$. Moreover, an object is said to be 4-connected if any of its pixels can be reached from any other pixel of the same object by traversing via 4-connected pixel pairs. An 8-connected object is defined in a similar manner.

\section{Boundary tracking}

An algorithm similar to edge following is known as boundary tracing ${ }^{[12]}$. The boundary image is formed as follows. For every pixel in the binary image, if the pixel is different from the pixel to its right or the pixel below it, then it is considered to be a part of the boundary image.

\section{Peak detection}

Starting with any boundary pixel, the image boundary is followed in a direction indicated by the direction flag ${ }^{[14]}$. Depending on the direction chosen at each step, the direction flags are appropriately modified. The points at which the vertical direction flag toggles are called peaks or valleys. Figure 5 shows a boundary of the palm image obtained from the binary image.

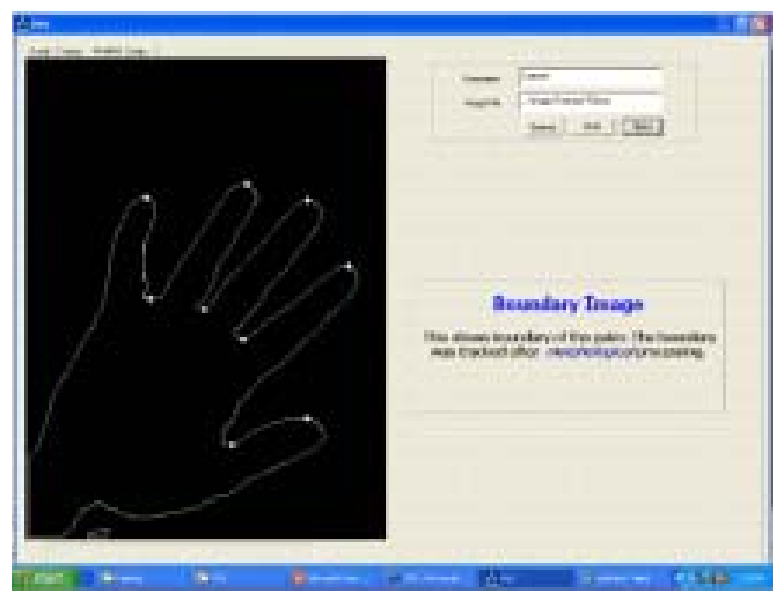

Fig.5 Palm boundary image 


\subsection{Orientation of the palm image}

PVS claims to be an orientation-independent palm verification system. In order to achieve this, it is necessary to align the captured image along a standard direction. This requires that we determine two points on the palm that define a standard direction and can be extracted from any palm image ${ }^{[4,5,12]}$. The inclination of the line joining these two points is the angle by which the palm image has to be rotated. This rotation of the image ensures orientation independence ${ }^{[13,14]}$. Figure 6 shows a boundary of the palm image obtained from the binary image.

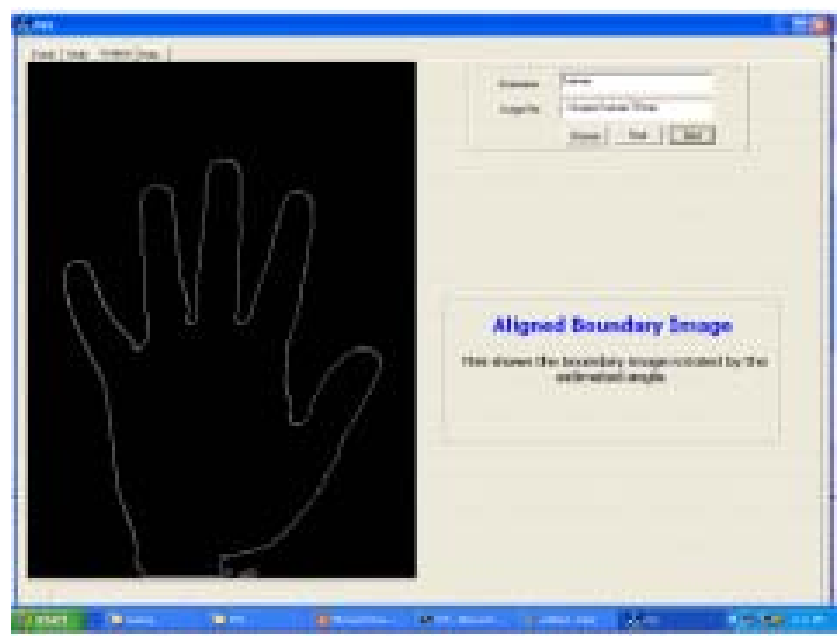

Fig.6 Aligned palm boundary

\subsection{Extraction of hand features}

The binary image as shown in Fig.7 is used to compute significant hand geometry features. A total of 12 hand geometry features were used (Fig.8); 4 finger lengths and 8 finger widths ( 2 widths per figure). Thus, the hand geometry of every hand image is characterized by a feature vector of length $1 \times 12$.

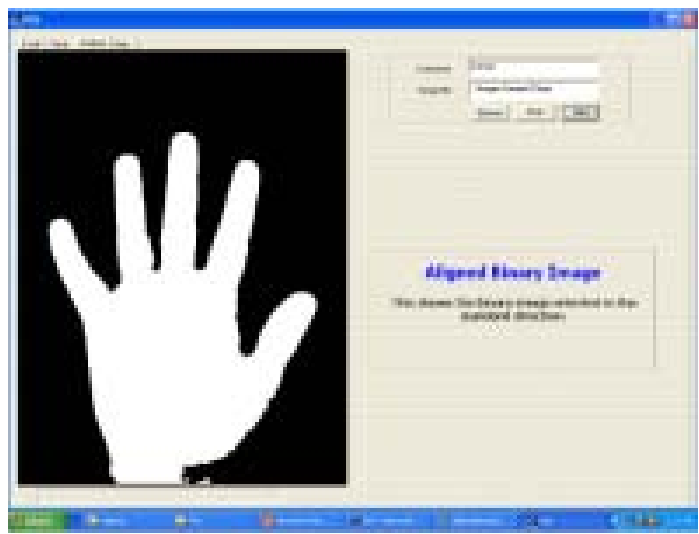

Fig.7 Aligned binary palm image

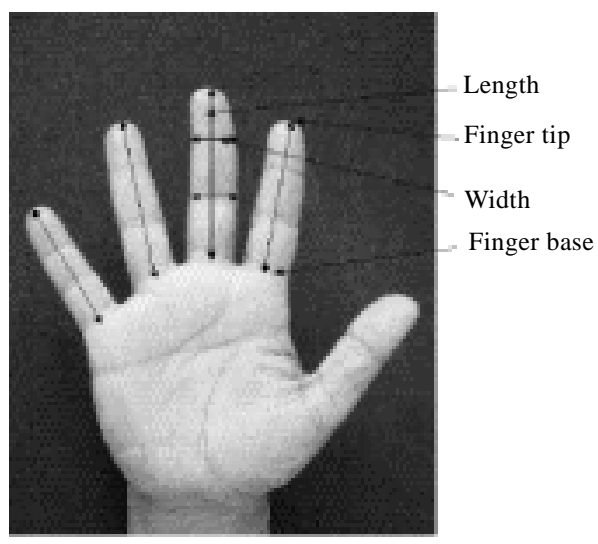

Fig.8 Hand geometry feature extraction

\subsection{Extraction of Palm features}

Figure 9 shows the aligned grayscale image from which the region of interest (ROI) is extracted ${ }^{[9]}$.

\subsubsection{Normalization}

The extracted palmprint images are normalized to have pre-specified mean and variance. The normalization is used 
to reduce the possible imperfections in the image due to sensor noise and non-uniform illumination. The method for normalization employed is as follows.

Let the gray level at $(x, y)$, in a palmprint image be represented by $I(x, y)$. The mean and variance of image, $f$ and $r$, respectively, can be computed from the gray levels of the pixels. Aligned grayscale image is shown in Fig.9.

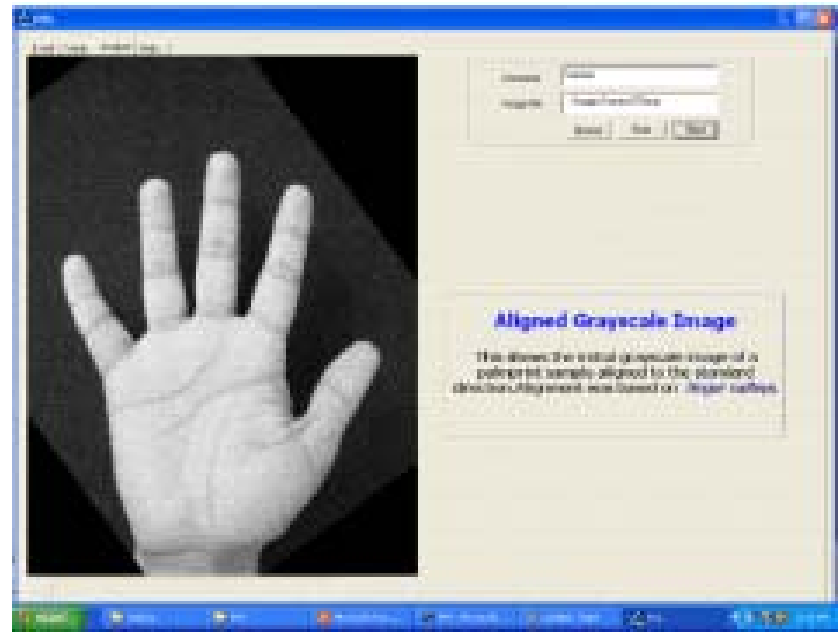

Fig.9 Aligned grayscale palm image

The normalized image, $I^{\prime}(x, y)$ is computed using the pixel-wise operations as follows

$$
I^{\prime}(x, y)=\left\{\begin{array}{l}
\phi_{d}+\lambda, \text { if } I(x, y)>\phi \\
\phi_{d}-\lambda, \text { otherwise }
\end{array}\right.
$$

where

$$
\lambda=\sqrt{\frac{\rho_{d}\{I(x, y)-\phi\}^{2}}{\rho}}
$$

where $\phi_{d}$ and $\rho_{d}$ are the desired values for mean and variance respectively. These values are pre-tuned according to the image characteristics, i.e., $I(x, y)$. In all our experiments, the values of $\phi_{d}$ and $\rho_{d}$ were fixed to 100 .

Figure 10 shows a typical palmprint image before and after the normalization.
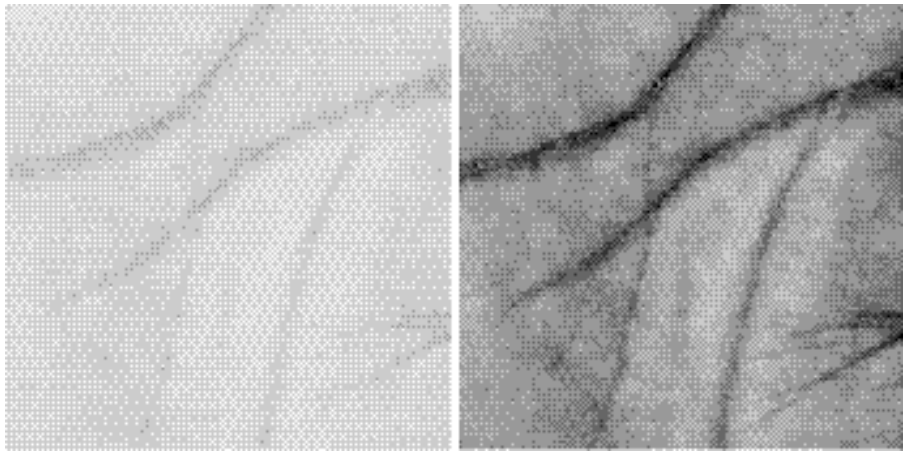

Fig.10 Extracted and normalized ROI

\subsubsection{Filtering}

The palmprint pattern is mainly made up of palm lines, i.e., principal lines and creases. Line feature matching is reported to be powerful and is of high accuracy in palmprint verification ${ }^{[13]}$. However, it is very difficult to 
accurately characterize these palm lines, i.e., their magnitude and direction, in noisy images. Therefore, a robust but simple method is used here. Line features from the normalized palmprint images are detected using four line detectors or directional masks. Each of these masks can detect lines oriented at $0 \mathrm{deg}, 90 \mathrm{deg}, 45 \mathrm{deg}$ and $135 \mathrm{deg}$. The spatial extent of these masks was empirically fixed as $9 \times 9$. Thus four filtered images, i.e. $I_{1}(x, y), I_{2}(x, y), I_{3}(x, y)$ and $I_{4}(x, y)$ are used to generate a final image $I_{f}(x, y)$ by

$$
I_{f}(x, y)=\max \left\{I_{1}(x, y), I_{2}(x, y), I_{3}(x, y), I_{4}(x, y)\right\}
$$

The resultant image represents the combined directional map of palmprint image. If $(x, y)$ is characterized by a set of localized features, i.e., standard deviation, and is used for verification, $I_{f}(x, y)$ is divided into a set $n$ blocks and the standard deviation of the gray-levels in each of these overlapping blocks is used to form the feature vector.

$$
v_{\text {palm }}=\left\{\sigma_{1}, \sigma_{2}, \ldots, \sigma_{n}\right\}
$$

where $\sigma_{i}$ is the standard deviation in the $i^{\text {th }}$ overlapping block.

Figure 11 shows the line features in the enhanced ROI.
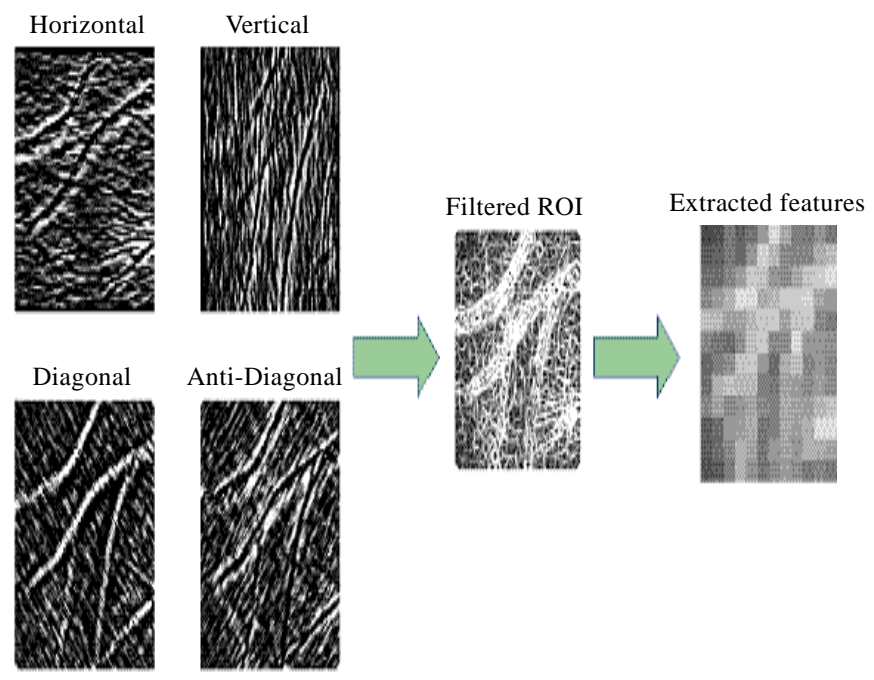

Fig.11 Palm feature extraction

\subsection{Matching criterion}

A two level threshold ${ }^{[14-16]}$ is used for the matching of both hand geometry and palm features.

\subsubsection{Hand geometry feature matching}

Hand geometry features are used for trivial rejection. The threshold for each feature is fixed at $70 \%$, and at least 10 out of the 12 features should meet this criterion for a successful match.

\subsubsection{Palm feature matching}

The standard deviation of each block is compared one-to-one with a threshold of $10 \%$. If the action of the blocks that satisfies the above test is more than $75 \%$, the two palm prints are said to match.

\section{Experimental Analysis}

The experimental analysis involves two steps.

I. Enrollment phase

II. Verification phase

In the Enrollment phase, the database of about 50 users along with their palmprint features is built. 
The input to the palmprint verification system is 256 gray scale bitmap images. Processing involves enhancement, orientation followed by extraction of features from the input image. A binary image obtained during enhancement is used to extract hand geometry features. The enhanced gray scale image is used to extract the palm print features. The output is a feature vector extracted from the image and is inserted into the database.

In the Verification phase, a test data of about 30 users were taken. Features were extracted from an input image; Input to palmprint verification system is 256 gray scale bitmap images along with the claimant's identifier. Processing involves enhancement, orientation followed by extraction of features from the input image and matching them with the corresponding claimant's palmprint image features from the database. The output of palmprint verification system is the indication of either match or mismatch is displayed. A slippery pair of statistics known as false rejection rate (FRR) and false acceptance rate represents the measure of experimental results (FAR). The sample results are shown in Fig.12 for the various thresholds.

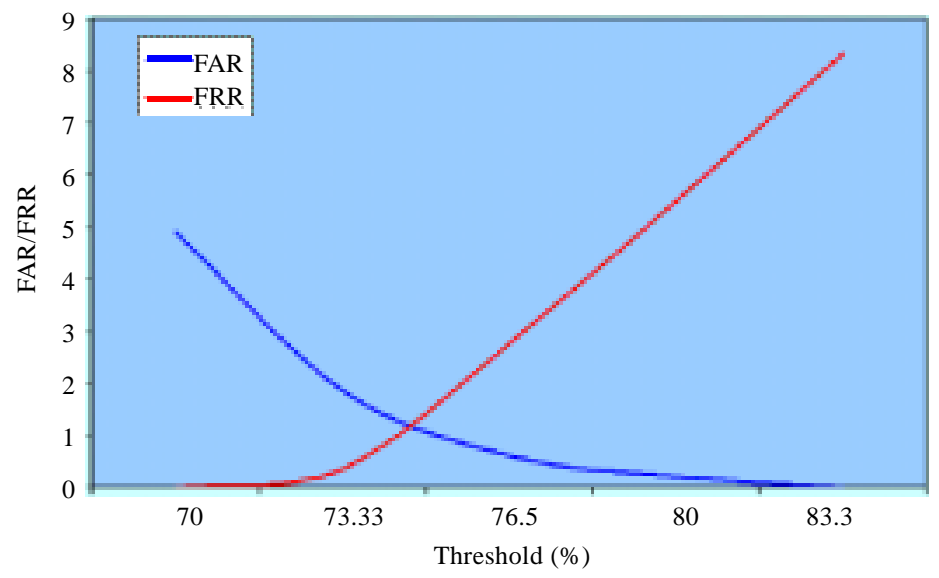

Fig.12 FAR/FRR versus threshold graph

\subsection{System test report}

\subsubsection{Common biometric measures}

\section{False rejection rate $(\mathrm{FRR})$}

This is a measure of the inconvenience caused due to false alarms when genuine users are not authenticated. This is a ratio of the number of rejected true claims to the total number of true claims, expressed in percentage ${ }^{[15,17]}$.

\section{FRR driver}

$$
\mathrm{FRR}=\frac{\text { Number of rejected client claims }}{\text { Total number of client accesses }} \text {. }
$$

The software module was developed to evaluate FRR of PVS on a palmprint database. The program enrolls a user with one of his palm images and tries to verify his identity with all his other images. This is repeated with different thresholds. The results are then tabulated and plotted.

\section{False acceptance rate (FAR)}

This is a measure of the ease with which a person can be impersonated. This is the ratio of the number of accepted false claims to the total number of false claims, expressed in percentage ${ }^{[15]}$.

$$
\mathrm{FRR}=\frac{\text { Number of accepted imposter claims }}{\text { Total number of imposter accesses }} \text {. }
$$

\section{FAR driver}

A software module was written to evaluate FAR of PVS on a palmprint database. The program enrolls a user with 
one of his palm images and tries to verify his identity with every palmprint image of every other user. This is repeated with different thresholds. The results are then tabulated and plotted as in Fig.12.

\section{Equal error rate (EER)}

EER is a point at which the FAR and FRR converge to a single point. This is a good choice for optimal threshold.

\section{Failure to enroll (FTE)}

This is the probability that the system fails to enroll a palm due to ergonomic or demographic peculiarities in the palm.

\section{Preventing database modification}

1. Use of binary format for storing palm features.

- Palm features are populated into a structure and written into a file.

2. Use of checksum with the extracted palm features.

- At the enrollment stage, a checksum is calculated by running a secret algorithm on the feature vector.

- At the verification stage, this value is recalculated and compared with the stored value.

Accuracy of the system is measured in terms of FAR and FRR for different thresholds (Tables 1 and 2) and the same is plotted (Figs.12 and 13) to get the optimal threshold for the system with low false acceptance and low false rejection rates.

Table 1 Values obtained for FAR and FRR against threshold

\begin{tabular}{cccccc}
\hline Threshold $(\%)$ & 70 & 73 & 76 & 80 & 83 \\
\hline FAR & 4.9 & 1.85 & 0.61 & 0 & 0 \\
FRR & 0 & 0 & 2.77 & 5.5 & 8.33 \\
\hline
\end{tabular}

Table 2 Values obtained for FRR and FAR

\begin{tabular}{cccccc}
\hline FAR & 4.9 & 1.85 & 0.61 & 0 & 0 \\
\hline FRR & 0 & 0 & 2.77 & 5.5 & 8.33 \\
\hline
\end{tabular}

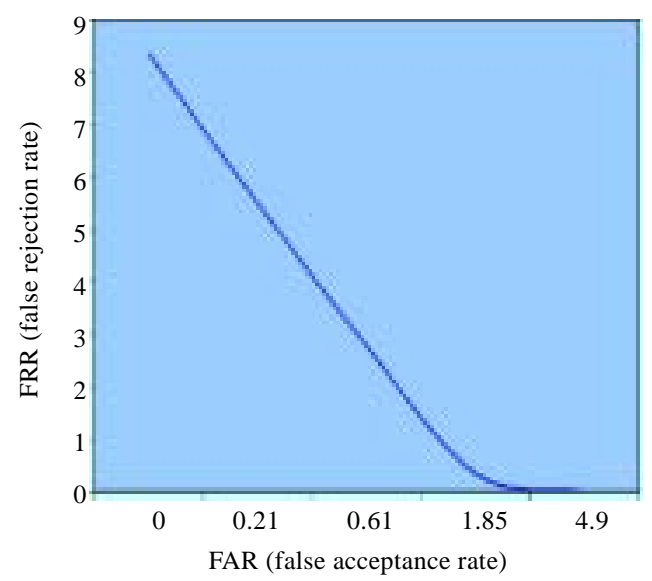

Fig.13 FAR versus FRR graph

\section{Conclusions}

The objective of this work is to investigate the integration of palm print and hand geometry features, and to achieve higher performance that may not be possible with single biometric indicator alone. The achieved results are significant since two biometric traits are derived from the same image unlike other bimodal biometric systems which require two different sensors/images. Also, the image resolution required is low when compared to other 
systems.

\section{References:}

[1] Duta N, Jain AK, Mardia KV. Matching of palmprint. Pattern Recognition Letters, 2002,23(2):477-485.

[2] Otsu N. A threshold selection method from gray-scale histogram. IEEE Trans. on System, Man, Cybernetics, 1978,8(1):62-66.

[3] Zhang D, Shu W. Two novel characteristics in palmprint verification: Datum point invariance and line feature matching. Pattern Recognition, 1999,32(4):691-702.

[4] NEC Automatic. Palmprint identification system. 2003. http://www.nectech.com/afis/download/PalmprintDtsht.q.pdf

[5] Kong WK, Zhang D. Palmprint texture analysis based on low-resolution images for personal authentication. In: Proc. of the ICPR-2002. Quebec City, 2002.

[6] Kumar A, Shen HC. Recognition of palmprints using wavelet-based features. In: Proc. of the Int'l Conf. on System, Cybernetics (SCI-2002). Orlando, 2002.

[7] Li W, Zhang D, Xu Z. Palmprint identification by Fourier transform. Int'l Journal Pattern Recognition and Artificial Intelligence, 2002,16(4):417-432.

[8] Shu W, Zhang D. Automated personal identification by palmprint. Optical Engineering, 1998,37(8):2359-2362.

[9] Chen J, Zhang C, Rong G. Palmprint recognition using crease. In: Proc. of the Int'1 Conf. on Image Process. 2001. $234-237$.

[10] Joshi DG, Rao YV, Kar S, Kumar V. Computer vision based approach to personal identification using finger crease pattern. Pattern Recognition, 1998,31(1):15-22.

[11] Kung Y, Lin SH, Fang M. A neural network based approach to face/palm recognition. In: Proc. of the Int'l Conf. on Neural Networks, 1995. 323-332.

[12] Han CC, Cheng HL, Lin CL, Fan KC. Personal authentication using palm-print features. Pattern Recognition, 2003,36:371-381.

[13] Sanchez-Reillo R, Sanchez-Avila C, Gonzales-Marcos A. Biometric identification through hand geometry measurements. IEEE Trans. on Pattern Analysis Machine Intelligence, 2000,22(10):1168-1171.

[14] Jain AK, Ross A, Pankarti S. A prototype hand geometry based verification system. In: Proc. of the 2nd Int'l Conf. on Audio Video Based Biometric Personal Authentication. Washington D.C., 1999. 166-171.

[15] Wu PS, Li M. Pyramid edge detection based on stack filter. Pattern Recognition Letters, 1997,18(4):239-248.

[16] Keegan JF. How can you tell if two line drawings are the same? Computer Graphics and Image Processing, 1997,6(1):90-92.

[17] You J, Li W, Zhang D. Hierarchical palmprint identification via multiple feature extraction. Pattern Recognition, 2002,35:847-859.

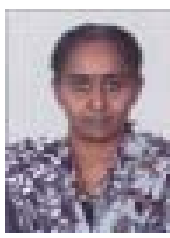

Dr G. Shobha, Asst. Professor, Department of Computer Science and Engineering, $\mathrm{R} \mathrm{V}$ College of Eng, Bangalore, India. She has got her doctoral degree for her thesis 'Knowledge Discovery in transactional Data base Systems' from Mangalore University. Her areas of interests are data mining, data base management system, and pattern recognition.

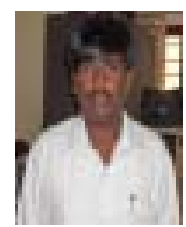

Dr M. Krishna, Asst. Professor, Department of Mechanical Engineering, Bangalore, India, has published 40 research papers in various International Journals and presented 75 research papers in various National/ International Conferences. $\mathrm{He}$ has been awarded various prestigious awards.

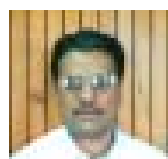

Dr S.C. Sharma is the Principal of $\mathrm{R} \mathrm{V}$ College of Engineering, Bangalore, India. $\mathrm{He}$ has won many prestigious awards and has around 350 journal publications and more 600 papers in the conference proceedings. He is the visiting professor of many prestigious universities. 